\title{
Outage performance of relay-assisted primary and secondary transmissions in cognitive relay networks
}

\author{
Feng Zhao ${ }^{1}$, Xiangqi Sun ${ }^{1}$, Hongbin Chen ${ }^{1 *}$ and Rongfang Bie ${ }^{2}$
}

\begin{abstract}
In this paper, a two-phase protocol for relay-assisted spectrum sharing in cognitive relay network is proposed. The primary system comprises a transmitter-receiver pair (PT-PR) and a decode-and-forward relay (Relay), while the secondary system comprises a transmitter-receiver pair (ST-SR). In the proposed protocol, the Relay assists the transmissions of the primary users as well as the secondary users where there is no direct link between the ST and the SR. Outage probabilities of the primary system and the secondary system are derived and verified through simulations. Compared with the protocol without cooperation, better outage performance of the primary system is achieved. Meanwhile, though the ST causes interference to the PR, the interference is compensated by the cooperation of the Relay. Moreover, the proposed protocol realizes the communications of the secondary users on the condition that the secondary transmission link is not ideal.
\end{abstract}

Keywords: Spectrum sharing; Cognitive relay network; Decode-and-forward relaying; Outage probability

\section{Introduction}

Traditional spectrum management policy, which is based on licensing of spectrum, allows the licensed users to access the frequency bands allocated to them. However, this policy cannot satisfy the increasing demand of users in wireless networks. The contradiction between the increasing demand and the limited spectrum resources compels us to improve the spectral efficiency.

For this purpose, some new technologies have been proposed. Cooperative diversity, as a new form of spatial diversity, was proposed to fight against channel fading and enhance throughput. The advantages of some cooperative diversity protocols have been studied in [1-3], and an adaptive cooperation diversity scheme has been studied in [4] which considered the mutual interference between the primary and secondary users. Cooperative relay, which uses intermediate entities to carry information

\footnotetext{
*Correspondence: chbscut@163.com

'Key Laboratory of Cognitive Radio and Information Processing, Guilin University of Electronic Technology, Ministry of Education, Guilin 541004, China

Full list of author information is available at the end of the article
}

from a source node to its destination node, is emerging as a promising technology to improve the reliability, throughput, and coverage areas of wireless networks. The most common relaying protocols are amplify-and-forward (AF) relaying and decode-and-forward (DF) relaying. The performance of DF relaying in spectrum sharing has been widely investigated in [5]. With the purpose of improving spectral efficiency, the concept of cognitive radio [6] was proposed by J. Mitola. In cognitive radio networks, secondary users transmit signals by detecting spectrum holes, or share the same frequency bands with primary users as long as the induced interference from secondary users to primary users is below a threshold [7]. The authors of [8] have studied a more general multi-user scenario which was extended from [7], and the authors of [9] have considered cooperative relaying for spectrum sensing in a twouser cognitive radio environment. Recently, cognitive relay network, which combines cognitive radio and cooperative relay, has attracted significant interests, and it is able to improve secondary users' transmission performance. The difference between cognitive relay networks and conventional relay networks has been studied in [10], and it showed that outage performance of cognitive relay networks is better than that of conventional relay networks. 
The authors of [11] have obtained a tight lower bound of the outage probability for underlay cognitive relay networks. A three-phase cognitive two-way relaying scenario has been analyzed in [12], and three different two-hop relaying protocols according to the amount of a prior knowledge have been investigated in [13]. Cognitive radio networks with opportunistic DF and uncoded DF-relaying schemes were considered and analyzed in [14]. When the achievements on the transmission performance of primary users increase, more and more attention has been paid to the transmission performance of secondary users. In [15], the authors have studied the capacity of the secondary users in cognitive relay networks, and proved that the reactive DF scheme is able to achieve the similar capacity of the proactive DF scheme. The performances of the secondary users in cognitive relay networks with the consideration of the primary users' interference have been analyzed in [16-18]. Especially, the authors of [17] have considered the outage performances of the primary and secondary systems on the condition that there exists a direct link between the secondary users. Power allocation has been applied to improve outage performance of the secondary system in [19].

Some other relevant researches have been done. Outage performances with relay selection in Rayleigh fading channels were studied in [20], and the full diversity order has been obtained for Rayleigh fading channels in [21,22]. Moreover, the authors of [23] have analyzed the impact of cooperation communications on the outage probability of cognitive radio networks over the Nakagami- $m$ fading channel and generalized the contributions in [21,22]. While more and more achievements on outage performance with perfect channel state information have been obtained, a lot of attention has been put into the one with imperfect channel state information. The transmissions of the secondary users will cause more interference at the primary users with imperfect channel state information. The authors of [24-28] have studied the transmission performances of the primary and secondary systems under imperfect channel state information.

Most of the existing research works focused on the separate performance of the primary system or the secondary system, and little research work has considered the performances of both primary and secondary systems. In view of this situation, we propose a protocol which employs a relay to help the transmissions of the primary and secondary systems contemporarily. With the cooperation of the relay, we try to decrease outage probability of the primary system, and compensate for the interference produced by the secondary users. Meanwhile, the relay assists the transmissions of the secondary users on the condition that there is no direct link between the secondary transmitter and the secondary receiver.
The rest of this paper is organized as follows: Section 2 describes the system model. In Section 3, we express the transmission signals in every transmission phase, and calculate outage probabilities of the primary and secondary systems. Section 4 presents the simulation results and discussions, and Section 5 concludes the paper.

\section{System model}

In cognitive radio systems, there is unlicensed spectrum for the transmissions of secondary users, whose priorities are lower than primary users. Secondary users are allowed to access the licensed spectrum in two cases: one is that secondary users utilize spectrum holes to transmit signals, and the other is that secondary users share licensed spectrum with primary users on the condition of causing tolerable harmful interference to primary users. In this case, it is impossible that there exists a portion of licensed spectrum for secondary users all the time. With the purposes of improving outage performance of the primary system and realizing communications of the secondary users without a direct link, we propose a spectrum sharing protocol where a relay assists the communications of the primary and secondary users, as depicted in Figure 1.

The primary system comprises a transmitter (PT), a receiver (PR), and a decode-and-forward relay (Relay), while the secondary system comprises a transmitter (ST) and a receiver (SR). The Relay assists the transmissions of the primary users and secondary users simultaneously. In the proposed system model, outage performance of the primary system benefits from the assistance of the Relay, and communications of the secondary users are achieved under the assistance of the Relay when there is no direct link between the ST and the SR.

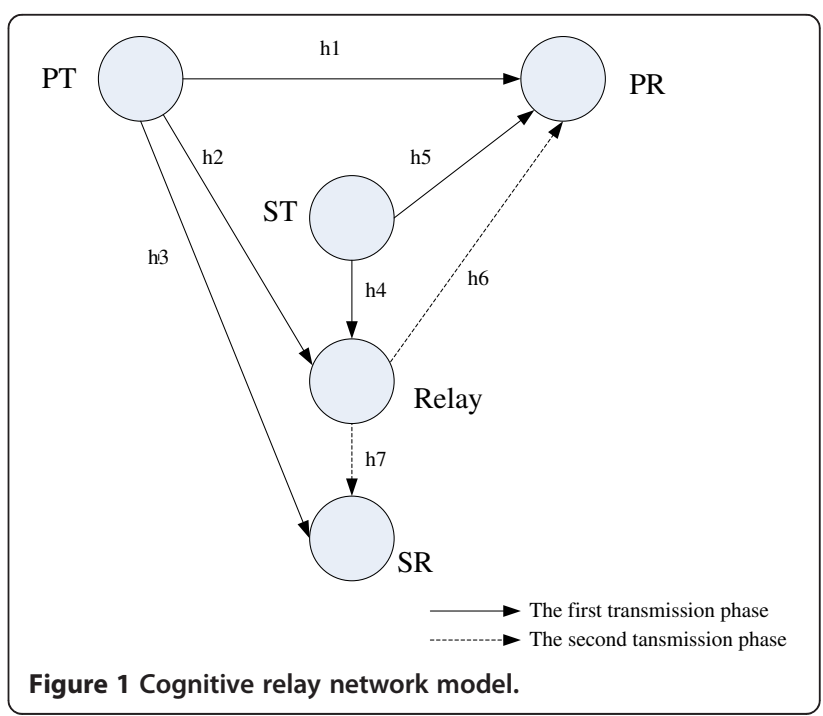


The whole transmission process is divided into two phases. In the first transmission phase, the PT and the ST transmit the primary and secondary signals, respectively. The PR, the Relay, and the SR receive the primary signal, and the Relay and the PR receive the secondary signal. Then, the primary signal and the secondary signal are decoded and superimposed at the Relay. In the second transmission phase, the $\mathrm{PR}$ and the $\mathrm{SR}$ receive a weighted linear composite signal which is transmitted by the Relay. At the PR, the secondary signal which is regarded as an interfering signal will be removed, and the primary signal will be restored if the PR is able to decode the secondary signal successfully. Then, the primary signal is retrieved by maximal-ratio combining (MRC) of the received signals from the two transmission phases at the PR. Similarly, the primary signal is regarded as an interfering signal and removed at the SR if the SR is successful to decode the primary signal, and the secondary signal is retrieved from the signal which is sent from the Relay.

All the channels are assumed to experience Rayleigh fading. The channel coefficients of the links PT $\rightarrow$ PR, $\mathrm{PT} \rightarrow$ Relay, $\mathrm{PT} \rightarrow \mathrm{SR}, \mathrm{ST} \rightarrow$ Relay, $\mathrm{ST} \rightarrow \mathrm{PR}$, Relay $\rightarrow$ $\mathrm{PR}$, and Relay $\rightarrow \mathrm{SR}$ are recorded by $h_{1}, h_{2}, h_{3}, h_{4}, h_{5}, h_{6}$, and $h_{7}$, respectively. Moreover, we assume $h_{i} \sim N\left(0, d_{i}^{-v}\right)$ $(i=1,2,3,4,5,6,7)$, which means that $h_{\mathrm{i}}$ is a circularly symmetric complex Gaussian random variable with variance $d_{i}^{-v}$. Here, $d_{i}$ represents the normalized distance between two nodes, and $v$ represents the path loss exponent. That is to say $d_{1}, d_{2}, d_{3}, d_{4}, d_{5}, d_{6}$, and $d_{7}$ denote the normalized distances between PT and PR, PT and Relay, PT and SR, ST and Relay, ST and PR, Relay and PR, and Relay and SR, respectively. This distance normalization is done with respect to the distance between PT and PR, i.e., $d_{1}=1$.

\section{Signal description and outage performance analysis}

\subsection{Outage performance of the proposed protocol} (scheme A)

In this subsection, outage performances of the primary system and the secondary system will be analyzed.

\subsubsection{Outage probability of the primary system}

In the first transmission phase, the PT transmits the primary signal $x_{p}$, and the PR, the Relay, and the SR receive the signal, respectively. The received signals of the PR, the Relay, and the SR are denoted by $y_{11}, y_{21}, y_{31}$, respectively, and we have

$$
y_{a 1}=\sqrt{P_{p}} h_{a} x_{p}+n_{a 1}
$$

where $a=1,2$, 3 . Here, $P_{p}$ represents transmission power of the PT, $h_{a}$ is a channel coefficient, and $n_{a 1} \sim N$ $\left(0, \sigma^{2}\right)$ is an additive white Gaussian noise with zero mean and variance $\sigma^{2}$. According to [7], the achievable rate between the PT and the Relay is calculated by

$$
R_{2}=\frac{1}{2} \log _{2}\left(1+\frac{P_{p}\left|h_{2}\right|^{2}}{\sigma^{2}}\right)=\frac{1}{2} \log _{2}\left(1+\frac{P_{p} \gamma_{2}}{\sigma^{2}}\right)
$$

Here, the value $\frac{1}{2}$ is due to the fact that the whole transmission process is divided into two transmission phases, and for ease of presentation, we record $\gamma_{j}=\left|h_{j}\right|^{2}$ $(j=1,2,3,4,5,6,7)$.

Likewise, the ST transmits the secondary signal $x_{s}$, and the signal is received by the Relay, as well as the PR. The received signals of the Relay and the $P R$ are recorded as $y_{41}$ and $y_{51}$, respectively, which can be expressed as

$$
y_{c 1}=\sqrt{P_{s}} h_{c} x_{s}+n_{c 1}
$$

where $c=4,5$. Here, $P_{s}$ represents transmission power of the ST, $h_{c}$ is a channel coefficient, and $n_{c 1} \sim N\left(0, \sigma^{2}\right)$ is an additive white Gaussian noise with zero mean and variance $\sigma^{2}$. The achievable rate between the ST and the Relay is recorded as $R_{4}$, and the one between the ST and the PR is recorded as $R_{5}$. They are calculated by

$$
R_{d}=\frac{1}{2} \log _{2}\left(1+\frac{P_{s} \gamma_{d}}{\sigma^{2}}\right)
$$

where $d=4,5$.

Then, the Relay decodes the signals sent from the PT and the ST, respectively. If the Relay is able to decode both $x_{p}$ and $x_{s}$, a composite signal $x_{R}$ is generated by linearly combining the regenerated signal $x_{p}$ with power $\alpha P_{r}$ and the secondary signal $x_{s}$ with power $(1-\alpha) P_{r}$, where $\alpha(0 \leq \alpha \leq 1)$ is the power allocation factor at the Relay, and $P_{r}$ is transmission power of the Relay. So, the composite signal is described by

$$
x_{R}=\sqrt{\alpha P_{r}} x_{p}+\sqrt{(1-\alpha) P_{r}} x_{s} .
$$

In the second transmission phase, the PR receives the signal which is sent from the Relay if the Relay decodes both $x_{p}$ and $x_{s}$ successfully. Otherwise, the Relay will keep silent. The received signal at the PR is recorded as $y_{12}$, which is given by

$$
y_{12}=\sqrt{\alpha P_{r}} h_{6} x_{p}+\sqrt{(1-\alpha) P_{r}} h_{6} x_{s}+n_{12},
$$

where $h_{6}$ is the channel coefficient of the link between the Relay and PR, and $n_{12} \sim N\left(0, \sigma^{2}\right)$ is an additive white Gaussian noise.

Then, the interference signal $\sqrt{(1-\alpha) P_{r}} h_{6} x_{s}$ will be removed from $y_{12}$ on the condition that the PR is successful to decode $x_{s}$. So, we have $y_{12}^{*}=\sqrt{\alpha P_{r}} h_{6} x_{p}+n_{12}$. 
Thus, MRC will be applied to combine $y_{11}$ and $y_{12}^{*}$. According to [7], the achievable rate is given by

$$
R_{11}^{\mathrm{MRC}}=\frac{1}{2} \log _{2}\left(1+\frac{P_{p} \gamma_{1}}{\sigma^{2}}+\frac{\alpha P_{r} \gamma_{6}}{\sigma^{2}}\right) .
$$

On the other condition that the PR fails to decode $x_{s}$, the secondary signal will be regarded as noise at the PR. Then, MRC will also be applied to combine $y_{11}$ and $y_{12}$. The achievable rate turns to be

$$
R_{12}^{\mathrm{MRC}}=\frac{1}{2} \log _{2}\left(1+\frac{P_{p} \gamma_{1}}{\sigma^{2}}+\frac{\alpha P_{r} \gamma_{6}}{(1-\alpha) P_{r} \gamma_{6}+\sigma^{2}}\right)
$$

For the primary system, there are two cases where the primary signal is transmitted successfully. The former is that $x_{p}$ is transmitted through the direct link from the PT to the PR on the condition that the Relay fails to decode either of $x_{p}$ or $x_{s}$. In this case, the achievable rate between the PT and the PR is given by $R_{1}=\log _{2}$ $\left(1+\frac{P_{p} \gamma_{1}}{\sigma^{2}}\right)$. The latter is that $x_{p}$ is transmitted from the relay-assisted link if the Relay decodes both $x_{p}$ and $x_{s}$ successfully. As mentioned, the former case includes three sub-cases, they are listed as follows: First, $x_{p}$ is transmitted through the direct link on the condition that the Relay succeeds to decode $x_{p}$ and fails to decode $x_{s}$. Second, $x_{p}$ is transmitted through the direct link on the condition that the Relay fails to decode $x_{p}$ and succeeds to decode $x_{s}$. Third, $x_{p}$ is transmitted through the direct link on the condition that the Relay fails to decode both $x_{p}$ and $x_{s}$.

The latter case includes two sub-cases, and they are listed as follows: One case is that the Relay succeeds to decode both $x_{p}$ and $x_{s}$, but the PR fails to decode $x_{s}$. Then, the primary signal $x_{p}$ is transmitted through the relay-assisted link. The other case is that the Relay succeeds to decode both $x_{p}$ and $x_{s}$, and the PR decodes $x_{s}$ successfully. Then, the primary signal $x_{p}$ is transmitted through the relay-assisted link.

The transmissions will be interrupted when the achievable rate is low than the target rate. So, outage probability of the primary system with target rate $R_{\mathrm{pt}}$ and $R_{\mathrm{st}}$ for the primary and secondary systems respectively, is given by

$$
P_{\mathrm{out}}^{p}=1-\left[\begin{array}{l}
P_{r}\left\{R_{2}>R_{\mathrm{pt}}\right\} P_{r}\left\{R_{4}<R_{\mathrm{st}}\right\} P_{r}\left\{\frac{1}{2} R_{1}>R_{\mathrm{pt}}\right\} \\
+P_{r}\left\{R_{2}<R_{\mathrm{pt}}\right\} P_{r}\left\{R_{4}>R_{\mathrm{st}}\right\} P_{r}\left\{\frac{1}{2} R_{1}>R_{\mathrm{pt}}\right\} \\
+P_{r}\left\{R_{2}<R_{\mathrm{pt}}\right\} P_{r}\left\{R_{4}<R_{\mathrm{st}}\right\} P_{r}\left\{\frac{1}{2} R_{1}>R_{\mathrm{pt}}\right\} \\
+P_{r}\left\{R_{2}>R_{\mathrm{pt}}\right\} P_{r}\left\{R_{4}>R_{\mathrm{s}\}}\right\} P_{r}\left\{R_{5}>R_{\mathrm{st}}\right\} P_{r}\left\{R_{11}^{\mathrm{MRC}}>R_{\mathrm{pt}}\right\} \\
+P_{r}\left\{R_{2}>R_{\mathrm{pt}}\right\} P_{r}\left\{R_{4}>R_{\mathrm{st}}\right\} P_{r}\left\{R_{5}<R_{\mathrm{st}}\right\} P_{r}\left\{R_{12}^{\mathrm{MMC}}>R_{\mathrm{pt}}\right\}
\end{array}\right]
$$

According to [7], since $\gamma_{1} \sim \varepsilon(1)$ and $\gamma_{e}^{\sim} \varepsilon\left(d_{e}^{v}\right) \quad(e=2$, $4,5,6)$, which means $\gamma_{e}$ is an exponentially distributed random variable with mean $\frac{1}{d_{e}^{v}}$, we have

$$
P_{r}\left\{\frac{1}{2} R_{1}>R_{\mathrm{pt}}\right\}=P_{r}\left\{\gamma_{1}>\frac{\rho_{1} \sigma^{2}}{P_{p}}\right\}=\exp \left(-\frac{\rho_{1} \sigma^{2}}{P_{p}}\right),
$$

$$
P_{r}\left\{R_{2}>R_{\mathrm{pt}}\right\}=P_{r}\left\{\gamma_{2}>\frac{\rho_{1} \sigma^{2}}{P_{p}}\right\}=\exp \left(-d_{2}^{\nu} \frac{\rho_{1} \sigma^{2}}{P_{p}}\right)
$$

$$
P_{r}\left\{R_{4}>R_{\mathrm{st}}\right\}=P_{r}\left\{\gamma_{4}>\frac{\rho_{3} \sigma^{2}}{P_{s}}\right\}=\exp \left(-d_{4}^{\nu} \frac{\rho_{3} \sigma^{2}}{P_{s}}\right)
$$

$$
P_{r}\left\{R_{5}>R_{\mathrm{st}}\right\}=P_{r}\left\{\gamma_{5}>\frac{\rho_{3} \sigma^{2}}{P_{s}}\right\}=\exp \left(-d_{5}^{v} \frac{\rho_{3} \sigma^{2}}{P_{s}}\right)
$$

$$
\begin{aligned}
P_{r}\left\{R_{11}^{\mathrm{MRC}}>R_{\mathrm{pt}}\right\}= & P_{r}\left\{\frac{1}{2} \log _{2}\left(1+\frac{P_{p} \gamma_{1}}{\sigma^{2}}+\frac{\alpha P_{r} \gamma_{6}}{\sigma^{2}}\right)>R_{\mathrm{pt}}\right\} \\
= & \left\{P_{p} \gamma_{1}+\alpha P_{r} \gamma_{6}>\rho_{1} \sigma^{2}\right\} \\
= & 1-\left\{P_{p} \gamma_{1}+\alpha P_{r} \gamma_{6} \leq \rho_{1} \sigma^{2}\right\} \\
= & \exp \left(-\frac{\rho_{1} \sigma^{2}}{P_{p}}\right)+\left(-1+d_{6}^{v} \frac{P_{p}}{\alpha P_{r}}\right)^{-1} \\
& \times\left[\exp \left(-\frac{\rho_{1} \sigma^{2}}{P_{p}}\right)-\exp \left(-d_{6}^{v} \frac{\rho_{1} \sigma^{2}}{\alpha P_{r}}\right)\right],
\end{aligned}
$$

where $\rho_{1}=2^{2 R_{\mathrm{pt}}}-1$. Assuming $P_{r} \gg \sigma^{2}$, we have

$$
\begin{aligned}
P_{r}\left\{R_{12}^{\mathrm{MRC}}>R_{\mathrm{pt}}\right\} & \approx P_{r}\left\{\frac{1}{2} \log _{2}\left[1+\frac{P_{p} \gamma_{1}}{\sigma^{2}}+\frac{\alpha}{1-\alpha}\right]>R_{\mathrm{pt}}\right\} \\
& =P_{r}\left\{\gamma_{1}>\frac{\sigma^{2}}{P_{p}}\left(\rho_{1}-\frac{\alpha}{1-\alpha}\right)\right\} \\
& =\left\{\begin{array}{cc}
\exp \left[-\frac{\sigma^{2}}{P_{p}}\left(\rho_{1}-\frac{\alpha}{1-\alpha}\right)\right] & 0 \leq \alpha<\alpha \\
1 & \alpha \leq \alpha \leq 1
\end{array},\right.
\end{aligned}
$$

where $\hat{\alpha}=\frac{\rho_{1}}{1+\rho_{1}}$. Substituting (10) to (15) into (9), we have

$$
P_{\text {out }}^{p}=\left\{\begin{array}{cc}
P_{\text {out }}^{p, 1} & 0 \leq \alpha<\hat{\alpha} \\
P_{\text {out }}^{p, 2} & \alpha \hat{\alpha} \leq \alpha \leq 1
\end{array} .\right.
$$


Here,

$$
P_{\text {out }}^{p, 1}=1-\left\{\begin{array}{l}
\exp \left(-d_{2}^{v} \frac{\rho_{1} \sigma^{2}}{P_{p}}\right)\left(1-\exp \left(-d_{4}^{v} \frac{\rho_{3} \sigma^{2}}{P_{s}}\right)\right) \exp \left(-\frac{\rho_{1} \sigma^{2}}{P_{p}}\right) \\
+\left(1-\exp \left(-d_{2}^{v} \frac{\rho_{1} \sigma^{2}}{P_{p}}\right)\right) \exp \left(-d_{4}^{v} \frac{\rho_{3} \sigma^{2}}{P_{s}}\right) \exp \left(-\frac{\rho_{1} \sigma^{2}}{P_{p}}\right) \\
+\left(1-\exp \left(-d_{2}^{v} \frac{\rho_{1} \sigma^{2}}{P_{p}}\right)\right)\left(1-\exp \left(-d_{4}^{v} \frac{\rho_{3} \sigma^{2}}{P_{s}}\right)\right) \exp \left(-\frac{\rho_{1} \sigma^{2}}{P_{p}}\right) \\
+\exp \left(-d_{2}^{v} \frac{\rho_{1} \sigma^{2}}{P_{p}}\right) \exp \left(-d_{4}^{v} \frac{\rho_{3} \sigma^{2}}{P_{s}}\right) \exp \left(-d_{5}^{v} \frac{\rho_{3} \sigma^{2}}{P_{s}}\right) \\
\left(\exp \left(-\frac{\rho_{1} \sigma^{2}}{P_{p}}\right)+\left(-1+d_{6}^{v} \frac{P_{p}}{\alpha P_{r}}\right)^{-1} \exp \left(-d_{6}^{v} \frac{\rho_{1} \sigma^{2}}{\alpha P_{r}}\right)\left(\exp \left(\left(-1+d_{6}^{v} \frac{P_{p}}{\alpha P_{r}}\right) \frac{\rho_{1} \sigma^{2}}{P_{p}}\right)-1\right)\right. \\
+\exp \left(-d_{2}^{v} \frac{\rho_{1} \sigma^{2}}{P_{p}}\right) \exp \left(-d_{4}^{v} \frac{\rho_{3} \sigma^{2}}{P_{s}}\right)\left[1-\exp \left(-d_{5}^{v} \frac{\rho_{3} \sigma^{2}}{P_{s}}\right)\right] \exp \left(-\frac{\sigma^{2}}{P_{p}}\left(\rho_{1}-\frac{\alpha}{1-\alpha}\right)\right)
\end{array}\right\},
$$

and

$$
P_{\text {out }}^{p, 2}=1-\left\{\begin{array}{l}
\exp \left(-d_{2}^{v} \frac{\rho_{1} \sigma^{2}}{P_{p}}\right)\left(1-\exp \left(-d_{4}^{v} \frac{\rho_{3} \sigma^{2}}{P_{s}}\right)\right) \exp \left(-\frac{\rho_{1} \sigma^{2}}{P_{p}}\right) \\
+\left(1-\exp \left(-d_{2}^{v} \frac{\rho_{1} \sigma^{2}}{P_{p}}\right)\right) \exp \left(-d_{4}^{v} \frac{\rho_{3} \sigma^{2}}{P_{s}}\right) \exp \left(-\frac{\rho_{1} \sigma^{2}}{P_{p}}\right) \\
+\left(1-\exp \left(-d_{2}^{v} \frac{\rho_{1} \sigma^{2}}{P_{p}}\right)\right)\left(1-\exp \left(-d_{4}^{v} \frac{\rho_{3} \sigma^{2}}{P_{s}}\right)\right) \exp \left(-\frac{\rho_{1} \sigma^{2}}{P_{p}}\right) \\
+\exp \left(-d_{2}^{v} \frac{\rho_{1} \sigma^{2}}{P_{p}}\right) \exp \left(-d_{4}^{v} \frac{\rho_{3} \sigma^{2}}{P_{s}}\right) \exp \left(-d_{5}^{v} \frac{\rho_{3} \sigma^{2}}{P_{s}}\right) \\
\left(\exp \left(-\frac{\rho_{1} \sigma^{2}}{P_{p}}\right)+\left(-1+d_{6}^{v} \frac{P_{p}}{\alpha P_{r}}\right)^{-1} \exp \left(-d_{6}^{v} \frac{\rho_{1} \sigma^{2}}{\alpha P_{r}}\right)\left(\exp \left(\left(-1+d_{6}^{v} \frac{P_{p}}{\alpha P_{r}}\right) \frac{\rho_{1} \sigma^{2}}{P_{p}}\right)-1\right)\right. \\
+\exp \left(-d_{2}^{v} \frac{\rho_{1} \sigma^{2}}{P_{p}}\right) \exp \left(-d_{4}^{v} \frac{\rho_{3} \sigma^{2}}{P_{s}}\right)\left[1-\exp \left(-d_{5}^{v} \frac{\rho_{3} \sigma^{2}}{P_{s}}\right)\right]
\end{array}\right\} .
$$

\subsubsection{Outage probability of the secondary system}

In the first transmission phase, the SR receives the signal which is sent from the PT, and the received signal is given by $y_{31}=\sqrt{P_{p}} h_{3} x_{p}+n_{31}$. The achievable rate between the PT and the SR is $R_{3}=\frac{1}{2} \log _{2}\left(1+\frac{P_{p} \gamma_{3}}{\sigma^{2}}\right)$. In the second transmission phase, the SR receives the signal $x_{R}$ which is sent from the Relay on the condition that the Relay succeeds to decode $x_{p}$ and $x_{s}$. We record the received signal as $y_{22}$, which is given by

$$
\begin{aligned}
y_{22} & =h_{7} x_{R}+n_{22} \\
& =\sqrt{\alpha P_{r}} h_{7} x_{p}+\sqrt{(1-\alpha) P_{r}} h_{7} x_{s}+n_{22} .
\end{aligned}
$$

Meanwhile, if the SR decodes the primary signal $x_{p}$ successfully, the component $\sqrt{\alpha P_{r}} h_{7} x_{p}$ will be removed from $y_{22}$ as an interfering signal. So we have $y_{22}^{*}=$ $\sqrt{(1-\alpha) P_{r}} h_{7} x_{s}+n_{22}$. The achievable rate between the Relay and the SR is calculated by

$$
R_{7}=\frac{1}{2} \log _{2}\left(1+\frac{(1-\alpha) P_{r} \gamma_{7}}{\sigma^{2}}\right)
$$

For the secondary system, the transmissions will be interrupted except the only case where the Relay decodes both $x_{p}$ and $x_{s}$ successfully, and the SR decodes $x_{p}$ successfully. So outage probability of the secondary system with the target rates $R_{\mathrm{pt}}$ and $R_{\mathrm{st}}$ is given by

$$
P_{\text {out }}^{s}=1-P_{r}\left\{R_{2}>R_{\mathrm{pt}}\right\} P_{r}\left\{R_{3}>R_{\mathrm{pt}}\right\} P_{r}\left\{R_{4}>R_{\mathrm{st}}\right\} P_{r}\left\{R_{7}>R_{\mathrm{st}}\right\} .
$$


Since $\gamma_{f} \sim \varepsilon\left(d_{f}^{v}\right)(f=3,7)$, which means an exponentially distributed random variable with mean $\frac{1}{d_{f}^{t}}$, we have

$$
\begin{aligned}
& P_{r}\left\{R_{3}>R_{\mathrm{pt}}\right\}=P_{r}\left\{\gamma_{3}>\frac{\rho_{1} \sigma^{2}}{P_{p}}\right\}=\exp \left(-d_{3}^{\nu} \frac{\rho_{1} \sigma^{2}}{P_{p}}\right), \\
& P_{r}\left\{R_{7}>R_{\mathrm{st}}\right\}=P_{r}\left\{\gamma_{7}>\frac{\rho_{3} \sigma^{2}}{(1-\alpha) P_{r}}\right\}=\exp \left(-d_{7}^{v} \frac{\rho_{3} \sigma^{2}}{(1-\alpha) P_{r}}\right) .
\end{aligned}
$$

So, substituting (11), (12), (20), and (21) into (19), we have

$$
\begin{aligned}
P_{\text {out }}^{s}= & 1-\exp \left(-d_{2}^{v} \frac{\rho_{1} \sigma^{2}}{P_{p}}\right) \exp \left(-d_{3}^{\nu} \frac{\rho_{1} \sigma^{2}}{P_{p}}\right) \\
& \times \exp \left(-d_{4}^{v} \frac{\rho_{3} \sigma^{2}}{P_{s}}\right) \exp \left(-d_{7}^{\nu} \frac{\rho_{3} \sigma^{2}}{(1-\alpha) P_{r}}\right) .
\end{aligned}
$$

\subsection{Outage performance of the protocol without cooperation (scheme B)}

The system without cooperation comprises of a transmitterreceiver pair (PT-PR), and the primary signal is transmitted through the direct link from the PT to the PR. Outage probability of the primary system is given by

$$
P_{\text {out }}^{o}=P_{r}\left\{R_{1}<R_{\mathrm{pt}}\right\}=1-\exp \left(-\frac{\sigma^{2}}{P_{p}} \rho_{2}\right) .
$$

where $\rho_{2}=2^{R_{\mathrm{pt}}}-1$. This protocol will be compared with our proposed protocol in Section 4.

\section{Simulation results and discussions}

The MATLAB tool is used to simulate the outage probabilities. The theoretical and numerical results for outage probabilities of the primary system and the secondary system versus the power allocation factor $\alpha$ in the proposed protocol (scheme A) are shown in Figure 2, and the results of the primary system's outage probability in the protocol without cooperation (scheme B) are also plotted for comparison. The topological structure of the cognitive relay network is constructed like this: the PT, PR, ST, SR, and Relay are collinear. In the twodimensional plane, the PT and the PR are located at the points $(0,0)$ and $(1,0)$, respectively. The Relay moves on the positive $X$-axis. The ST is located at the midpoint of the PT and the Relay, while the SR is located at the midpoint of the Relay and the PR. In this topology, we let $R_{\mathrm{pt}}=R_{\mathrm{st}}=1, v=4, P_{p}=P_{s}=P_{r}=10 \mathrm{~W}, \sigma^{2}=1, d_{1}=1, d_{3}=$ $\frac{1}{2}\left(1+d_{2}\right), d_{4}=\frac{1}{2} d_{2}, d_{5}=1-\frac{1}{2} d_{2}, d_{6}=\left|1-d_{2}\right|, d_{7}=\frac{1}{2}$ $\left|1-d_{2}\right|$, and the values of $d_{2}$ are selected as $0.5,0.8$, and

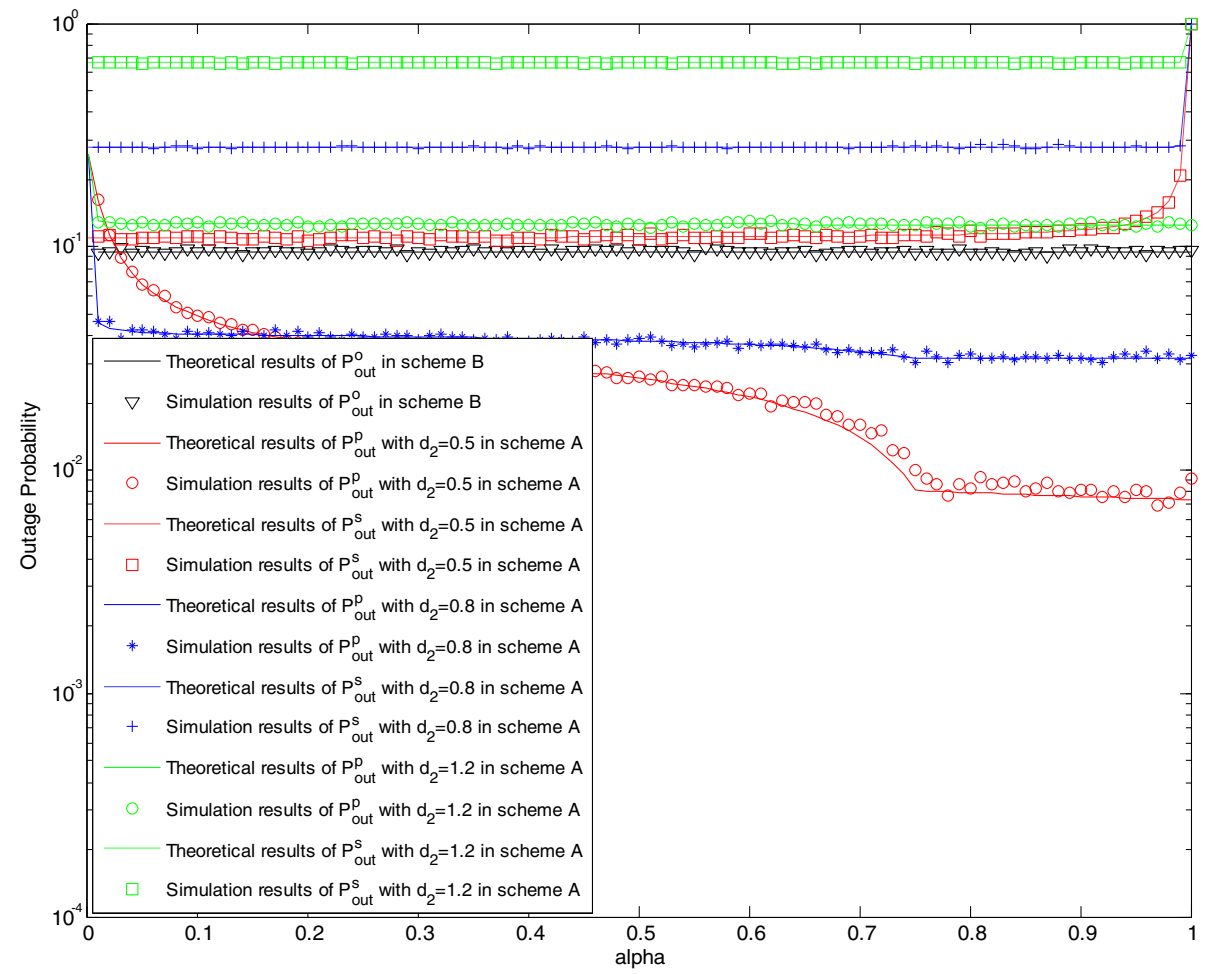

Figure 2 Outage probability comparison in case of $d_{2}=0.5, d_{2}=0.8$, and $d_{2}=1.2$. 
1.2. It is easy to find that the theoretical results match well with the numerical results from Figure 2. For $d_{2}=0.5$ and 0.8 , outage probabilities of the primary system in scheme A are lower than those in scheme B when $\alpha \geq \alpha^{*} \approx 0.05$, i.e., $P_{\text {out }}^{p}<P_{\text {out }}^{o}$. It means that outage performance of the primary system benefits from the cooperation of the Relay. For $d_{2}=1.2$, outage probability in scheme $\mathrm{A}$ is a little higher than that in scheme $\mathrm{B}$, i.e., $P_{\text {out }}^{p}>P_{\text {out }}^{o}$. Through analysis and discussions, we know that the Relay is able to assist the transmissions of the primary and secondary users, and interference will be introduced to the primary users when the Relay assists the transmissions of the secondary users. For $d_{2}=1.2$, the distance between the PT and Relay is far away from that between the PT and PR, and the benefit of cooperation from the Relay is not obvious compared with the introduced interference.

From Figure 2, we also find that the secondary users are able to communicate smoothly with the cooperation of the Relay when $d_{2}=0.5,0.8,1.2$. Moreover, we can get that there exist two extreme cases, i.e., $\alpha=0$ and $\alpha=$ 1 . The former $(\alpha=0)$ corresponds to the protocol where the primary signal is only transmitted from the PT to the PR, and the whole transmission power of the Relay is used to transmit the secondary signal, which is regarded as an interference signal at the PR. It is easy to get that outage probability of the secondary system is satisfactory, but outage probability of the primary system is unsatisfactory. With the increasing of $\alpha$, outage performance of the primary system becomes better, and there is no obvious effect on outage performance of the secondary system. That is to say, outage performance of the primary system in the proposed protocol is better than that in the protocol where the Relay assists the transmissions of the secondary users only. The latter $(\alpha=1)$ means the protocol where the Relay helps the transmissions of the primary users only. In this protocol, though outage probability of the primary system is low than that in the proposed protocol, outage probability of the secondary system is equal to 1 , which means that the ST cannot communicate with the SR. So, we are able to better balance outage performances of the primary system and the secondary system in the proposed protocol.

It is easy to find that outage probabilities of the primary system and the secondary system are affected obviously by the variation of $d_{2}$. So we show the effects of $d_{2}$ on outage performances of the primary and secondary systems in Figure 3. Here, we adopt the same topological structure as in Figure 2, and let $R_{\mathrm{pt}}=R_{\mathrm{st}}=1, v=4, P_{p}=$ $P_{s}=P_{r}=10 \mathrm{~W}, \sigma^{2}=1, \alpha=0.5$. According to the results from Figure $2, d_{2}$ is set to be less than $d_{1}$, which means that the distance between the PT and the Relay is less than that between the PT and the PR. From Figure 3, it is obvious that the outage probability of the primary

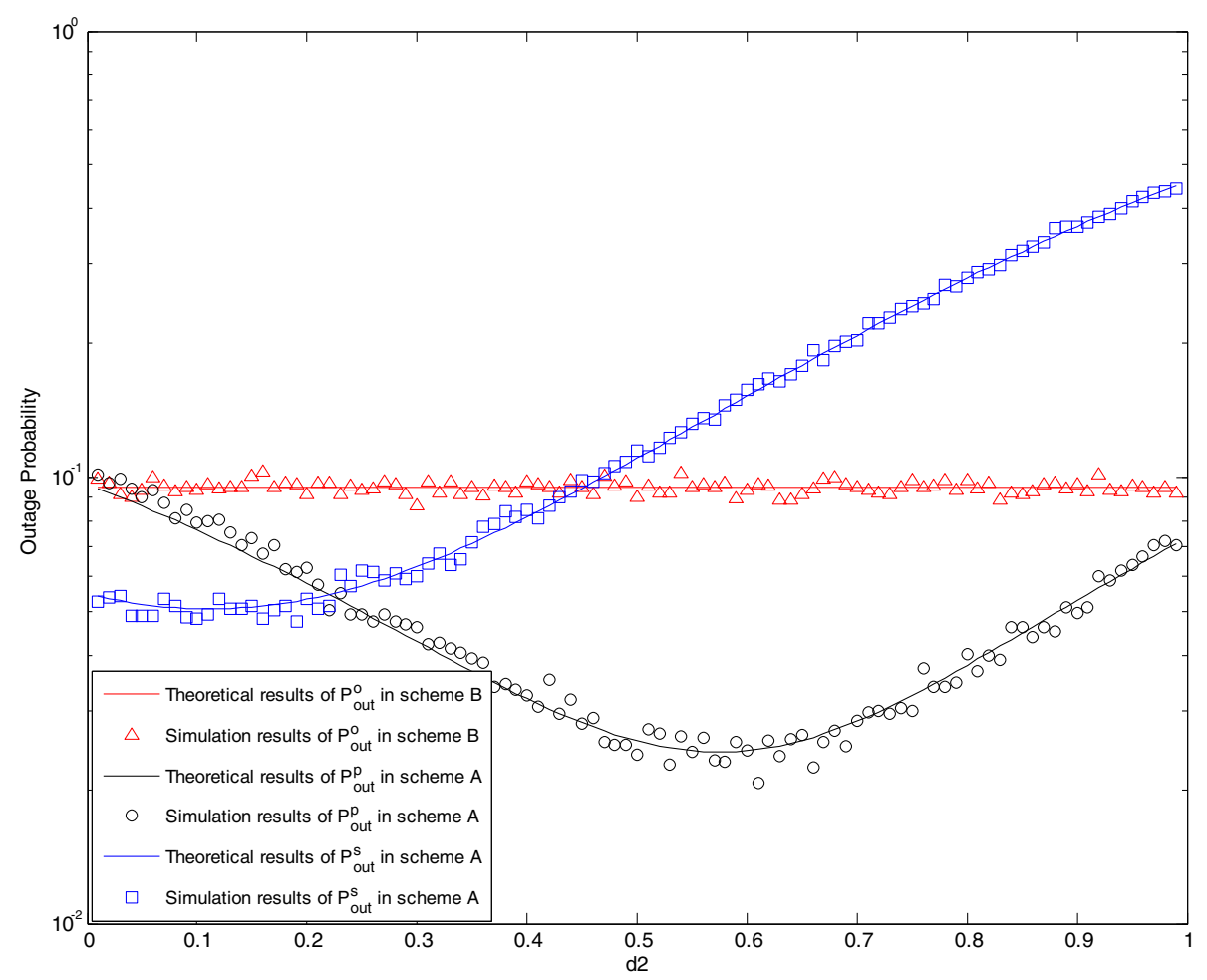

Figure 3 Outage probability versus $d_{2}$. 
system in scheme $\mathrm{A}$ is less than or equal to that in scheme B. When $d_{2} \leq d_{2}^{*} \approx 0.5$, the outage probability of the primary system decreases with the increasing of $d_{2}$, but it increases with the increasing of $d_{2}$ when $d_{2}>d_{2}^{*} \approx$ 0.5 . From the results, we know that outage performance of the primary system will be better if the distance between the PT and Relay is less than that between the PT and PR, and it coincides with the analysis in Figure 2. Moreover, outage probability of the secondary system increases with the increasing of $d_{2}$.

Then, we consider the effects of transmission power on the outage performances. We show outage probabilities of the primary and secondary systems versus $P_{r}, P_{p}$, and $P_{s}$ in Figures 4, 5, and 6, respectively. Here, we adopt the same topological structure as in Figure 2, and let $R_{\mathrm{pt}}=R_{\mathrm{st}}=$ $1, v=4, \sigma^{2}=1, \alpha=0.5$. Meanwhile, when one of $P_{r} P_{p}$, and $P_{s}$ is regarded as a variable, the other two parameters are set to $10 \mathrm{~W}$. From Figure 4, it is easy to get that outage probabilities of the primary and secondary systems decrease with the increasing of $P_{r}$. The reason for these results is that the Relay has the ability to assist the transmissions of the primary users as well as the secondary users, but there exists the mutual interference between the primary and secondary users. With the increasing of $P_{r}$, both of the cooperative capability and mutual interference become more and more, so outage probabilities of the primary and secondary systems tend to be stable.
In Figure 5, we show the effect of $P_{p}$ on the outage performances. Through analysis, we know that $P_{\text {out }}^{p}, P_{\text {out }}^{s}$, and $P_{\text {out }}^{o}$ decrease with the increasing of $P_{p}$ from (16), (22), and (23), and they meet the simulation results in Figure 5. Because $P_{p}$ is the transmission power of the $\mathrm{PT}$, the outage probability of the primary system will be better with the increasing of $P_{p}$. For the secondary users, the decoding of the primary signal benefits from the increase of $P_{p}$, so the outage probability of the secondary system also decreases with the increasing of $P_{p}$.

The effects of $P_{s}$ on outage performances are shown in Figure 6. It can be seen that both $P_{\text {out }}^{p}$ and $P_{\text {out }}^{s}$ decrease with the increasing of $P_{s}$. For the secondary system, $P_{\text {out }}^{s}$ decreases with the increasing of $P_{s}$ from (22). Even though, there is not an obvious diminution of $P_{\text {out }}^{s}$ from Figure 6 . The reason is that there is not a direct link between the ST and the SR, which means that transmissions of the secondary users must be assisted by the Relay. So the effects of $P_{s}$ cannot be reflected obviously on $P_{\text {out }}^{s}$. For the primary system, the interference caused by the secondary users will become larger, but the increase of $P_{s}$ also contributes to the decoding of secondary signal at the PR and Relay. Then, it helps the cooperative transmissions of the primary signal at the Relay. That is to say, the increase of $P_{s}$ will cause larger interference as well as better cooperation for the primary system, and the benefit of cooperation is far more than

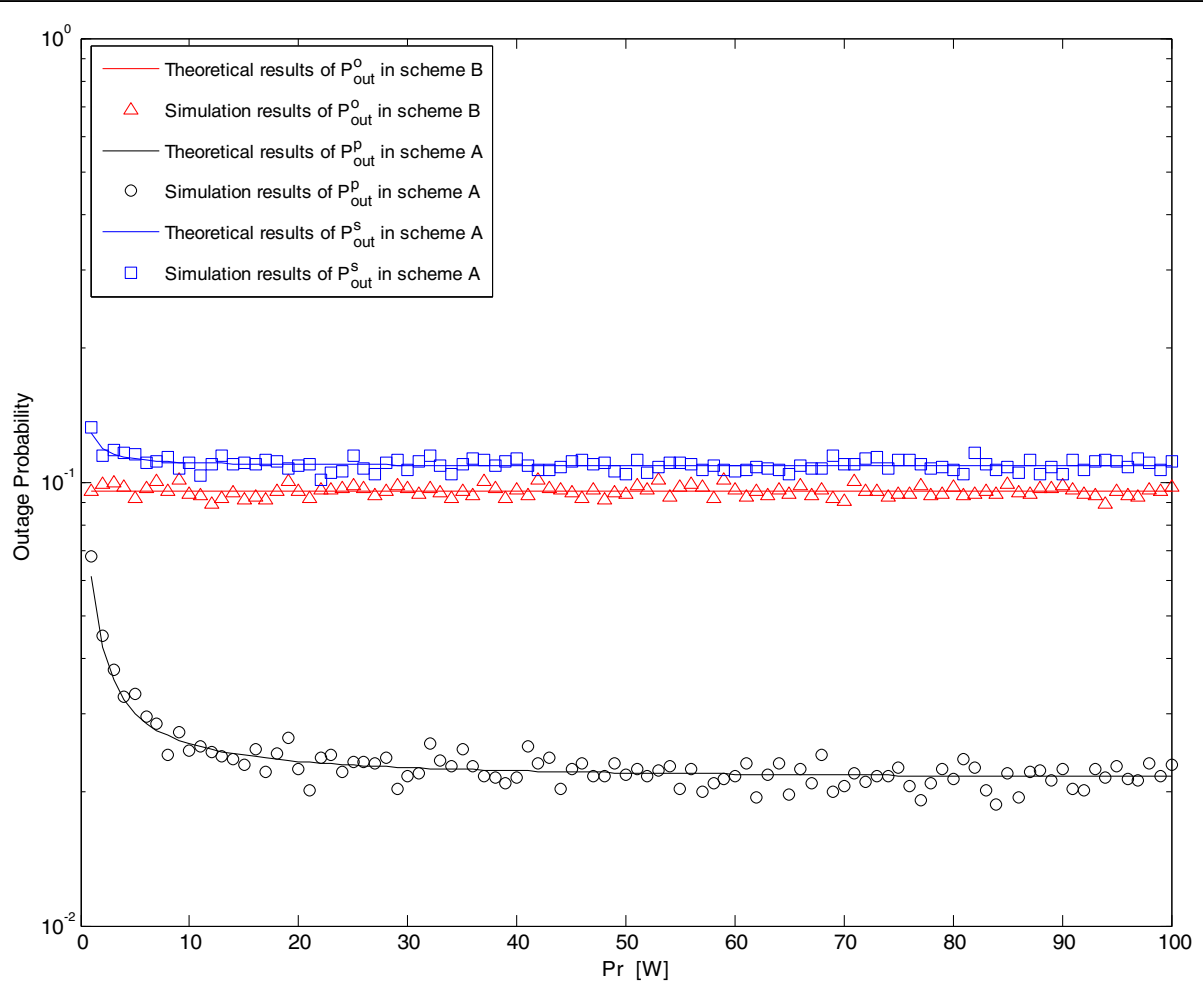

Figure 4 Outage probability versus $P_{r}$. 


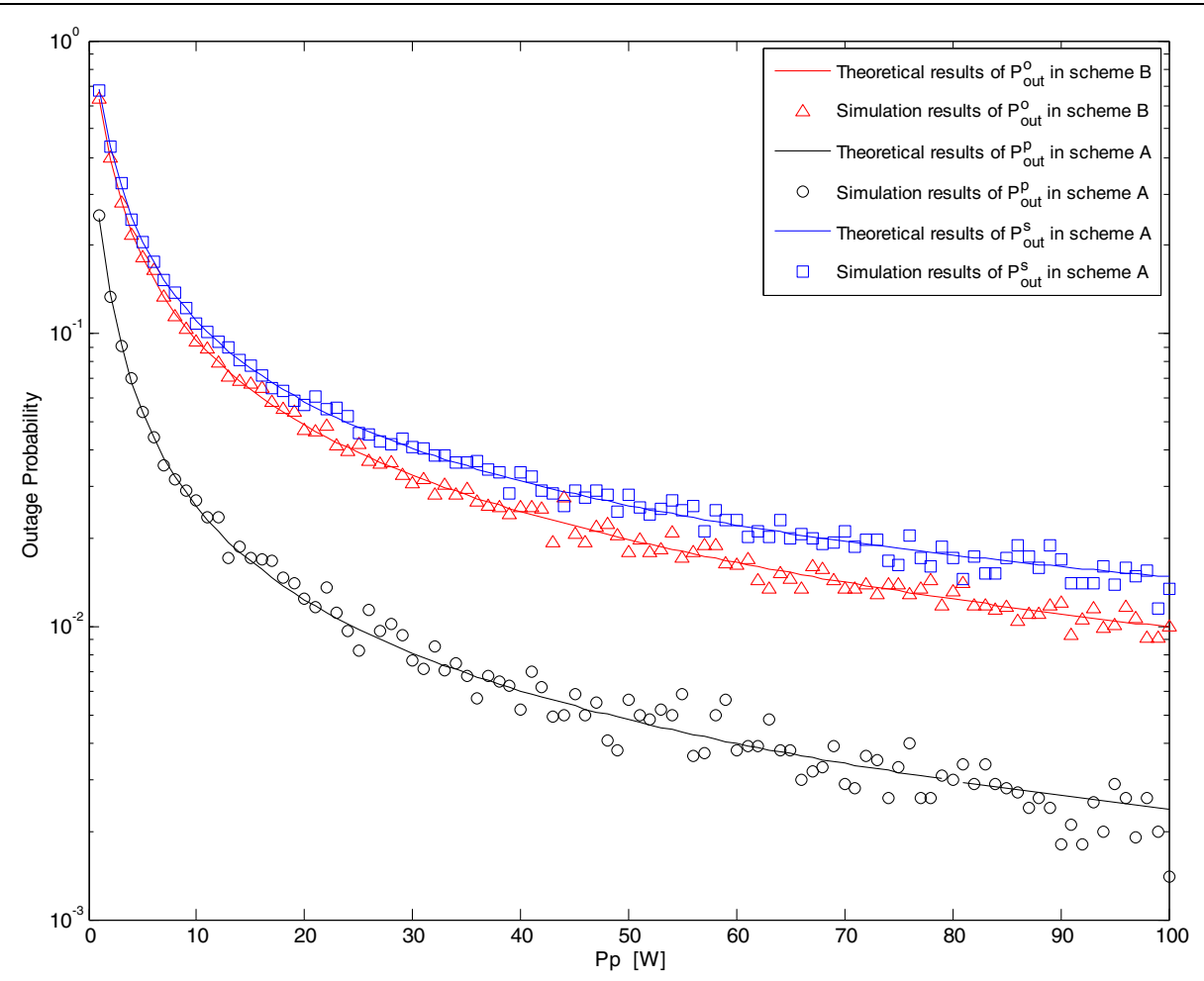

Figure 5 Outage probability versus $P_{p}$.

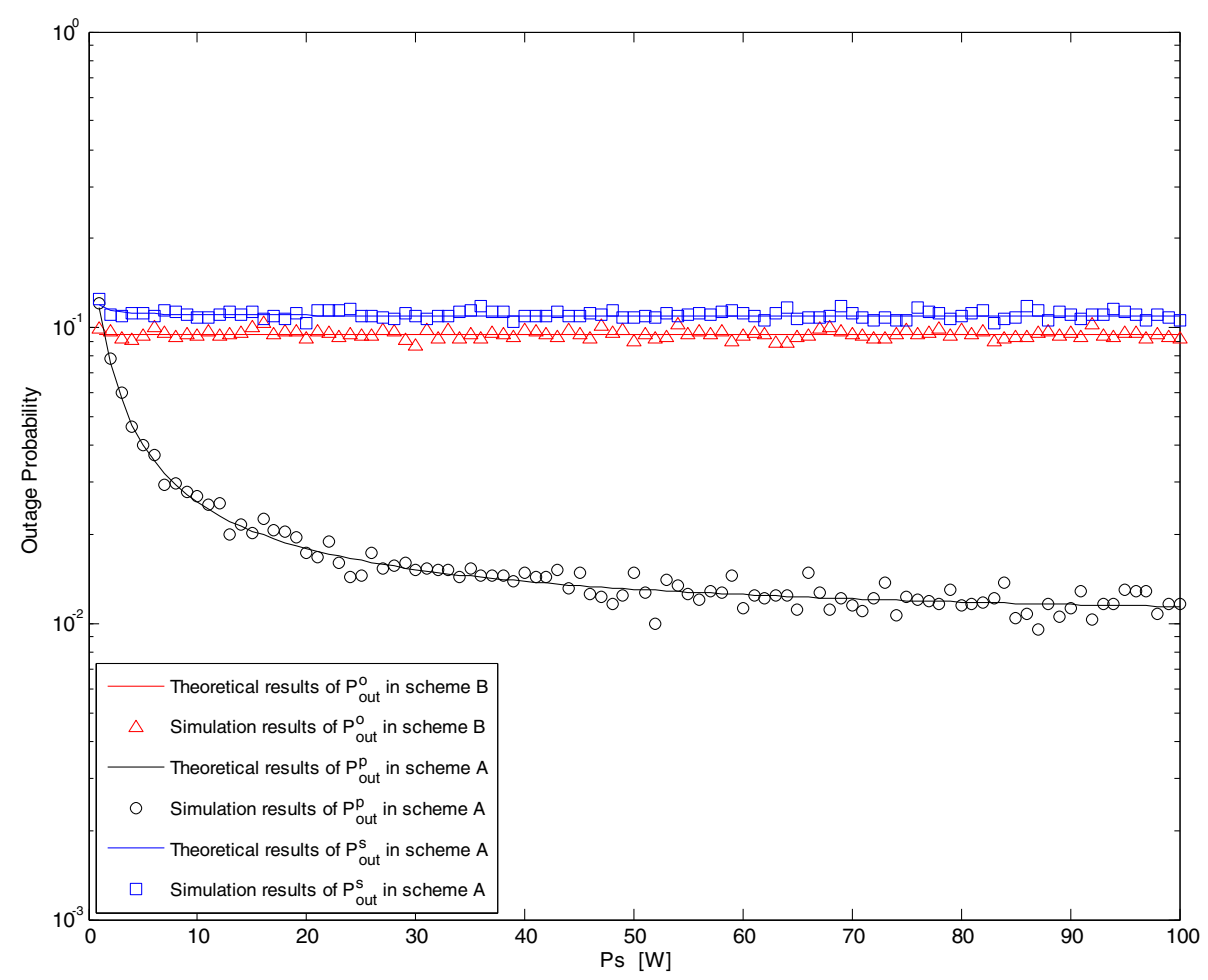

Figure 6 Outage probability versus $P_{s}$. 
the interference when $P_{s}$ increases. So there is an obvious improvement on outage performance of the primary system with the increasing of $P_{s}$.

\section{Conclusions}

In this paper, a relay-assisted spectrum sharing protocol in cognitive relay networks has been presented. In the proposed protocol, better outage performance of the primary users is achieved, and communications of the secondary users without a direct link are realized under the assistance of a decode-and-forward relay.

\section{Competing interests}

The authors declare that they have no competing interests.

\section{Acknowledgements}

We would like to thank the National Natural Science Foundation of China $(61162008,61172055)$, the Guangxi Natural Science Foundation (2011GXNSFB018072, 2013GXNSFGA019004), the Key Project of Chinese Ministry of Education (212131), the Foundation of Department of Education of Guangxi Province (201202ZD045, 201202ZD046), the Open Research Fund of Guangxi Key Lab of Wireless Wideband Communication \& Signal Processing $(12103,12106)$, and the Director Fund of Key Laboratory of Cognitive Radio and Information Processing (Guilin University of Electronic Technology), Ministry of Education, China (2013ZR02).

\section{Author details}

${ }^{1}$ Key Laboratory of Cognitive Radio and Information Processing, Guilin University of Electronic Technology, Ministry of Education, Guilin 541004, China. ${ }^{2}$ College of Information Science and Technology, Beijing Normal University, Beijing 100875, China.

Received: 11 March 2014 Accepted: 26 March 2014 Published: 16 April 2014

\section{References}

1. TE Hunter, S Sanayei, A Nosratinia, Outage analysis of coded cooperation. IEEE Trans. Inf. Theory 52(2), 375-391 (2006)

2. Y Zou, B Zheng, J Zhu, Outage analysis of opportunistic cooperation over Rayleigh fading channels. IEEE Trans. Wirel. Commun. 8(6), 3077-3085 (2009)

3. Y Zou, B Zheng, W Zhu, An opportunistic cooperation scheme and its BER analysis. IEEE Trans. Wirel. Commun. 8(9), 4492-4497 (2009)

4. Y Zou, J Zhu, B Zheng, Y Yao, An adaptive cooperation diversity scheme with best-relay selection in cognitive radio networks. IEEE Trans. Signal Process. 58(10), 5438-5445 (2010)

5. V Asghari, S Aïssa, End-to-end performance of cooperative relaying in spectrum sharing systems with quality of service requirements. IEEE Trans. Veh. Technol. 60(6), 2656-2668 (2011)

6. J Mitola, Cognitive radio: making software radios more personal. IEEE Pers. Commun. 6(4), 13-18 (1999)

7. Y Han, A Pandharipande, SH Ting, Cooperative decode-and-forward relaying for secondary spectrum access. IEEE Trans. Wirel. Commun. 8(10), 4945-4950 (2009)

8. Y Han, SH Ting, A Pandharipande, Cooperative spectrum sharing protocol with secondary user selection. IEEE Trans. Wirel. Commun. 9(9), 5438-5445 (2010)

9. G Ganesan, Y Li, Cooperative spectrum sensing in cognitive radio-part I: two user networks. IEEE Trans. Wirel. Commun. 6(6), 2204-2213 (2007)

10. J Lee, $\mathrm{H}$ Wang, JG Andrews, D Hong, Outage probability of cognitive relay networks with interference constraints. IEEE Trans. Wirel. Commun. 10(2), 390-395 (2011)

11. L Luo, P Zhang, G Zhang, J Qin, Outage performance for cognitive relay networks with underlay spectrum sharing. IEEE Commun. Lett. 15(7), 710-712 (2011)

12. Q Li, SH Ting, A Pandharipande, Y Han, Cognitive spectrum sharing with two-way relaying systems. IEEE Trans. Veh. Technol. 60(3), 1233-1240 (2011)

13. W Chang, Cognitive radios for preserving primary outage performance over two-hop relay channels. IEEE Commun. Lett. 16(8), 1176-1179 (2012)
14. D Li, Cognitive relay networks: opportunistic or uncoded decode-andforward relaying. IEEE Trans. Veh. Technol. 63(3), 1486-1491 (2014)

15. S Sagong, J Lee, D Hong, Capacity of reactive DF scheme in cognitive relay networks. IEEE Trans. Wirel. Commun. 10(10), 3133-3138 (2011)

16. J Si, Z Li, H Huang, J Chen, R Gao, Capacity analysis of cognitive relay networks with the PU's interference. IEEE Commun. Lett. 16(12), 2020-2023 (2012)

17. P Yang, L Luo, J Qin, Outage performance of cognitive relay networks with interference from primary user. IEEE Commun. Lett. 16(10), 1695-1698 (2012)

18. H Huang, Z Li, J Si, R Gao, Outage analysis of underlay cognitive multiple relays networks with a direct link. IEEE Commun. Lett. 17(8), 1600-1603 (2013)

19. X Kang, Optimal power allocation for fading cognitive multiple access channels: a two-user case. IEEE Wirel. Commun. Lett. 2(6), 683-686 (2013)

20. X Zhang, Z Yan, Y Gao, W Wang, On the study of outage performance for cognitive relay networks with the Nth best-relay selection in Rayleigh-fading channels. IEEE Wirel. Commun. Lett 2(1), 110-113 (2013)

21. TQ Duong, VNQ Bao, GC Alexandropoulos, H Zepernick, Cooperative spectrum sharing networks with AF relay and selection diversity. Electron. Lett. 47(20), 1149-1151 (2011)

22. J Hong, B Hong, TW Ban, W Choi, On the cooperative diversity gain in underlay cognitive radio systems. IEEE Trans. Commun. 60(1), 209-219 (2012)

23. TQ Duong, DB Costa, TA Tsiftsis, C Zhong, A Nallanathan, Outage and diversity of cognitive relaying systems under spectrum sharing environments in Nakagami-m fading. IEEE Commun. Lett. 16(12), 2075-2078 (2012)

24. J Chen, J Si, Z Li, H Huang, On the performance of spectrum sharing cognitive relay networks with imperfect CSI. IEEE Commun. Lett. 16(7), 1002-1005 (2012)

25. SH Safavi, M Ardebilipour, S Salari, Relay beamforming in cognitive two-way networks with imperfect channel state information. IEEE Wirel. Commun. Lett. 1(4), 344-347 (2012)

26. X Zhang, J Xing, Z Yan, Y Gao, W Wang, Outage performance study of cognitive relay networks with imperfect channel knowledge. IEEE Commun. Lett. 17(1), 27-30 (2013)

27. X Gong, A Ispas, G Dartmann, G Ascheid, Power allocation and performance analysis in spectrum sharing systems with statistical CSI. IEEE Trans. Wirel. Commun. 12(4), 1819-1831 (2013)

28. K Hamdi, MO Hasna, A Ghrayeb, KB Letaief, Opportunistic spectrum sharing in relay-assisted cognitive systems with imperfect CSI. IEEE Trans. Veh. Technol. (2013). doi:10.1109/TVT.2013.2289878

doi:10.1186/1687-1499-2014-60

Cite this article as: Zhao et al:: Outage performance of relay-assisted primary and secondary transmissions in cognitive relay networks. EURASIP Journal on Wireless Communications and Networking 2014 2014:60.

\section{Submit your manuscript to a SpringerOpen ${ }^{\circ}$ journal and benefit from:}

- Convenient online submission

- Rigorous peer review

- Immediate publication on acceptance

- Open access: articles freely available online

- High visibility within the field

- Retaining the copyright to your article

Submit your next manuscript at $\gg$ springeropen.com 